\title{
On Lecacheux's family of quintic polynomials
}

\author{
By Akinari $\operatorname{HoshI}^{*)}$ and Masakazu KoshiBA ${ }^{* *}$ \\ (Communicated by Kenji FukAyA, M.J.A., Dec. 14, 2020)
}

\begin{abstract}
Kida, Rikuna and Sato [6] developed a classification theory for Brumer's quintic polynomials via Kummer theory arising from associated elliptic curves. We generalize their results to elliptic curves associated to Lecacheux's quintic $F_{20}$-polynomials instead of Brumer's quintic $D_{5}$-polynomials.
\end{abstract}

Key words: Lecacheux's quintic polynomial; Kummer theory; elliptic curves.

1. Introduction. Let $K$ be a field with char $K \neq 2,5$ and $C_{n}$ be the cyclic group of order $n$. Let $D_{5} \simeq C_{5} \rtimes C_{2}$ be the dihedral group of order 10 and $F_{20} \simeq C_{5} \rtimes C_{4}$ be the Frobenius group of order 20. Let $K(s, t)$ be the rational function field over $K$ with two variables $s, t$. Brumer's quintic polynomial $\operatorname{Bru}(t, s ; X)$ is defined to be

(1) $\operatorname{Bru}(t, s ; X):=X^{5}+(t-3) X^{4}-(t-s-3) X^{3}$

$$
+\left(t^{2}-t-2 s-1\right) X^{2}+s X+t \in K(s, t)[X] \text {. }
$$

The polynomial $\operatorname{Bru}(t, s ; X)$ is $K$-generic for $D_{5}$, namely (i) the Galois group of $\operatorname{Bru}(t, s ; X)$ over $K(s, t)$ is isomorphic to $D_{5}$; and (ii) every $D_{5}$-Galois extension $L / M, \# M=\infty, M \supset K$, can be obtained as $L=\operatorname{Spl}_{M}(\operatorname{Bru}(b, a ; X))$, the splitting field of $\operatorname{Bru}(b, a ; X)$ over $M$, for some $a, b \in M$ (see Jensen, Ledet and Yui [4, Theorem 2.3.5]).

Kida, Rikuna and Sato [6] studied Brumer's quintic $\operatorname{Bru}(t, s ; X)$ via Kummer theory arising from elliptic curves. The splitting field $\operatorname{Spl}_{K(s, t)}(\operatorname{Bru}(t, s ; X))$ contains the unique quadratic subfield $K(s, t)\left(\sqrt{d_{t, s}}\right)$ where

(2) $d_{t, s}:=-4 s^{3}+\left(t^{2}-30 t+1\right) s^{2}$

$$
\begin{aligned}
& +2 t(3 t+1)(4 t-7) s \\
& -t\left(4 t^{4}-4 t^{3}-40 t^{2}+91 t-4\right) \in K(s, t) .
\end{aligned}
$$

In this paper, we study the case where $K=\mathbf{Q}$. We search elements $\alpha$ and $\beta$ in $\mathbf{Q}(s, t)$ such that the quadratic subfields of $\operatorname{Spl}_{\mathbf{Q}(s, t)}(\operatorname{Bru}(\beta, \alpha ; X))$ and of $\mathrm{Spl}_{\mathbf{Q}(s, t)}(\operatorname{Bru}(t, s ; X))$ coincide. According to Kida, Rikuna and Sato [6, Section 2], we restrict ourselves to treat the case $\beta=t$ and consider the equation

2010 Mathematics Subject Classification. Primary 11G05, 11R20, 12F20, $12 \mathrm{G} 05$.

*) Department of Mathematics, Niigata University, Niigata 950-2181, Japan.

**) Graduate School of Science and Technology, Niigata University, Niigata 950-2181, Japan.

$$
d_{t, s} u^{2}=d_{t, \alpha}
$$

Define

$$
d=d_{t, s}, x=-4 d \alpha, y=4 d^{2} u .
$$

Then we obtain the associated elliptic curve

(3) $E_{t, s}: y^{2}=x^{3}+d\left(t^{2}-30 t+1\right) x^{2}$

$$
\begin{aligned}
& -8 d^{2} t(3 t+1)(4 t-7) x \\
& -16 d^{3} t\left(4 t^{4}-4 t^{3}-40 t^{2}+91 t-4\right)
\end{aligned}
$$

to Brumer's quintic polynomial $\operatorname{Bru}(t, s ; X)$. This elliptic curve $E_{t, s}$ has an isogeny $\phi$ of degree 5 defined over $\mathbf{Q}(s, t)$. The 5-division polynomial of $E_{t, s}$ (see Silverman [8, Exercise 3.7]) has a quadratic factor $f_{2}(x)$ (see $[1$, Section 1]). Take a root $\theta$ of $f_{2}(x)=0$. Then we obtain a point $A \in E_{t, s}(\overline{\mathbf{Q}(s, t)})$ of order 5 with $x(A)=\theta$. Apply the Vélu formula [10] to $\langle A\rangle$ and take $E_{t, s}^{*}=E_{t, s} /\langle A\rangle$ as the image of $\phi$ (see Kida, Rikuna and Sato [6, Section 2]):

(4) $\quad E_{t, s}^{*}: y^{2}=x^{3}+d\left(t^{2}-30 t+1\right) x^{2}$

$$
\begin{gathered}
-8 d^{2}\left(26 t^{4}-310 t^{3}+327 t^{2}+315 t+26\right) x \\
+16 d^{3}\left(68 t^{6}-1120 t^{5}+3804 t^{4}+1760 t^{3}\right. \\
\left.+6929 t^{2}+1380 t+68\right) .
\end{gathered}
$$

After the specialization $\mathbf{Q}(s, t)^{2} \ni(s, t) \mapsto\left(s^{\prime}, t^{\prime}\right) \in$ $\mathbf{Q}^{2}$, we obtain that $\operatorname{Bru}\left(t^{\prime}, s^{\prime} ; X\right), E_{t^{\prime}, s^{\prime}}$ and $E_{t^{\prime}, s^{\prime}}^{*}$ are defined over $\mathbf{Q}$. After the specialization, for $s, t \in \mathbf{Q}$, we also write $\operatorname{Bru}(t, s ; X), E_{t, s}$ and $E_{t, s}^{*}$ which are defined over $\mathbf{Q}\left(\right.$ not $\mathbf{Q}(s, t)$ ). Let $\phi^{*}: E_{t, s}^{*} \rightarrow E_{t, s}$ be the dual isogeny of $\phi$. Then the quotient group $E_{t, s}(\mathbf{Q}) / \phi^{*}\left(E_{t, s}^{*}(\mathbf{Q})\right)$ is finite by weak Mordel-Weil theorem (see [8, Chapter VIII, Section 1]).

Definition 1.1 (Kida, Rikuna and Sato [6, page 694]). Let $s, t$ be rational numbers. For each Q-rational point $P=(x(P), y(P)) \in E_{t, s}(\mathbf{Q})$, Brumer's polynomial $\operatorname{Bru}(P ; X)$ with respect to $P$ is defined to be 


$$
\operatorname{Bru}(P ; X):=\operatorname{Bru}\left(t, \frac{x(P)}{-4 d} ; X\right)
$$

where $\operatorname{Bru}(t, s ; X)$ is Brumer's polynomial as in (1) and $d=d_{t, s}$ is given as in (2).

We remark that there exists a rational point $P_{0}=\left(-4 d s, 4 d^{2}\right) \in E_{t, s}(\mathbf{Q})$ and by the definition we have $\operatorname{Bru}\left(P_{0} ; X\right)=\operatorname{Bru}(t, s ; X)$.

Theorem 1.2 (Kida, Rikuna and Sato [6, Theorem 2.1]). Let $s, t$ be rational numbers. Let $E_{t, s}$ be the elliptic curve as in (3). Let $\operatorname{Bru}(P ; X)$ be Brumer's polynomial with respect to $P$ as in Definition 1.1 with the splitting field $\operatorname{Spl}_{\mathbf{Q}}(\operatorname{Bru}(P ; X))$ over $\mathbf{Q}$.

(i) For any $\mathbf{Q}$-rational point $P \in E_{t, s}(\mathbf{Q}), \operatorname{Bru}(P ; X)$ is reducible over $\mathbf{Q}$ if and only if $P \in \phi^{*}\left(E_{t, s}^{*}(\mathbf{Q})\right)$;

(ii) There exists a bijection between the following two finite sets

$\left\{\right.$ subgroup of order 5 in $\left.E_{t, s}(\mathbf{Q}) / \phi^{*}\left(E_{t, s}^{*}(\mathbf{Q})\right)\right\}$ and

$\left\{\operatorname{Spl}_{\mathbf{Q}}(\operatorname{Bru}(P ; X)) \mid P \in E_{t, s}(\mathbf{Q}) \backslash \phi^{*}\left(E_{t, s}^{*}(\mathbf{Q})\right)\right\}$.

The bijection is induced by the correspondence $E_{t, s}(\mathbf{Q}) \ni P \mapsto \operatorname{Spl}_{\mathbf{Q}}(\operatorname{Bru}(P ; X))$.

The aim of this paper is to generalize Theorem 1.2 to elliptic curves associated to Lecacheux's quintic $F_{20}$-polynomial $\operatorname{Lec}(p, r ; X)$ instead of Brumer's quintic $D_{5}$-polynomial $\operatorname{Bru}(t, s ; X)$.

Let $\mathbf{Q}(p, r)$ be the rational function field over $\mathbf{Q}$ with two variables $p, r$. Lecacheux's quintic polynomial $\operatorname{Lec}(p, r ; X)$ is defined to be

(5) $\operatorname{Lec}(p, r ; X):=X^{5}+\left(r^{2}\left(p^{2}+4\right)-2 p-\frac{17}{4}\right) X^{4}$

$$
\begin{aligned}
& +\left(3 r\left(p^{2}+4\right)+p^{2}+\frac{13}{2} p+5\right) X^{3} \\
& -\left(r\left(p^{2}+4\right)+\frac{11}{2} p-8\right) X^{2} \\
& +(p-6) X+1 \in \mathbf{Q}(p, r)[X] .
\end{aligned}
$$

The polynomial $\operatorname{Lec}(p, r ; X)$ is known to be Q-generic for $F_{20}$ (see [4, Theorem 2.3.6]).

We will define the elliptic curve $\mathcal{E}_{p, r}$ associated to Lecacheux's polynomial $\operatorname{Lec}(p, r ; X)$. Define

$$
\begin{aligned}
W_{p, r}:= & 16\left(p^{2}+4\right) r^{3}+4\left(p^{2}+4\right) r^{2} \\
& -4(19 p+41) r-16 p-199,
\end{aligned}
$$

$$
\begin{aligned}
D_{p, r}:= & \frac{W_{p, r}}{8}\left(\left(p^{4}+5 p^{2}+4\right)\right. \\
& \left.+p\left(p^{2}+3\right) \sqrt{p^{2}+4}\right) .
\end{aligned}
$$

The splitting field $\operatorname{Spl}_{\mathbf{Q}(p, r)}(\operatorname{Lec}(p, r ; X))$ contains the unique quadratic (resp. quartic) subfield $\mathbf{Q}(p, r)\left(\sqrt{p^{2}+4}\right)\left(\operatorname{resp.} \mathbf{Q}(p, r)\left(\sqrt{D_{p, r}}\right)\right)$ (see Hoshi and Miyake [2, Lemma 7.3 and Lemma 7.4]; $\operatorname{Lec}(p, r ; X)$ is $g_{p, r}^{F_{20}}(X)$ in [2]).

We search $\beta$ such that the quartic subfields of $\operatorname{Spl}_{\mathbf{Q}}(\operatorname{Lec}(p, \beta ; X))$ and of $\operatorname{Spl}_{\mathbf{Q}}(\operatorname{Lec}(p, r ; X))$ coincide. We consider the equation

$$
D_{p, r} u^{2}=D_{p, \beta} .
$$

Write $D=D_{p, r}$ and $W=W_{p, r}$. Then the above equation becomes

$$
W u^{2}=W_{p, \beta} .
$$

Define

$$
x:=4\left(p^{2}+4\right) W \beta, y:=2\left(p^{2}+4\right) W^{2} u .
$$

Then we get the associated elliptic curve

$$
\begin{aligned}
\mathcal{E}_{p, r}: y^{2}= & x^{3}+\left(p^{2}+4\right) W x^{2} \\
& -4(19 p+41)\left(p^{2}+4\right) W^{2} x \\
& -4\left(p^{2}+4\right)^{2}(16 p+199) W^{3}
\end{aligned}
$$

to Lecacheux's quintic polynomial $\operatorname{Lec}(p, r ; X)$.

The curve $\mathcal{E}_{p, r}$ has an isogeny $\nu$ of degree 5 defined over $\mathbf{Q}(p, r)$. We see that the 5-division polynomial of $\mathcal{E}_{p, r}$ (see Silverman [8, Exercise 3.7]) has the quadratic factor $f_{2}(x)$ (see $[1$, Section 1$]$ ). Take a root $\theta$ of $f_{2}(x)=0$. Then we obtain a point $A \in \mathcal{E}_{p, r}(\overline{\mathbf{Q}(p, r)})$ of order 5 with $x(A)=\theta, \mathcal{E}_{p, r}^{*}=$ $\mathcal{E}_{p, r} /\langle A\rangle$ as the image of $\nu$ and the dual isogeny $\nu^{*}$ : $\mathcal{E}_{p, r}^{*} \rightarrow \mathcal{E}_{p, r}$ of $\nu$ as in (4) (see also Kida, Rikuna and Sato [6, Section 2]):

$$
\begin{aligned}
\mathcal{E}_{p, r}^{*}: y^{2}= & x^{3}+\left(p^{2}+4\right) W x^{2} \\
& -4\left(p^{2}+4\right)\left(52 p^{2}-625 p+833\right) W^{2} x \\
& +4\left(p^{2}+4\right)^{2}\left(272 p^{2}-5000 p+21713\right) W^{3} .
\end{aligned}
$$

As in the case of Brumer's quintic, after the specialization $\mathbf{Q}(p, r)^{2} \ni(p, r) \mapsto(p, r) \in \mathbf{Q}^{2}$, we also write $\operatorname{Lec}(p, r ; X), \mathcal{E}_{p, r}$ and $\mathcal{E}_{p, r}^{*}$ for $p, r \in \mathbf{Q}$ which are defined over $\mathbf{Q}($ not $\mathbf{Q}(p, r))$.

Definition 1.3. Let $p, r$ be rational numbers. For each Q-rational point $P=(x(P), y(P)) \in$ $\mathcal{E}_{p, r}(\mathbf{Q})$, Lecacheux's polynomial $\operatorname{Lec}(P ; X)$ with respect to $P$ is defined to be 


$$
\operatorname{Lec}(P ; X):=\operatorname{Lec}\left(p, \frac{x(P)}{4\left(p^{2}+4\right) W} ; X\right)
$$

where $\operatorname{Lec}(p, r ; X)$ is Lecacheux's polynomial as in (5) and $W=W_{p, r}$ is given as in (6).

We note that there exists the point $Q_{0}=$ $\left(4 r\left(p^{2}+4\right) W, 2\left(p^{2}+4\right) W^{2}\right) \in \mathcal{E}_{p, r}(\mathbf{Q})$ and we have $\operatorname{Lec}\left(Q_{0} ; X\right)=\operatorname{Lec}(p, r ; X)$ by the definition.

The following is the main theorem of this paper:

Theorem 1.4. Let $p, r$ be rational numbers. Let $\mathcal{E}_{p, r}$ be the elliptic curve as in (7). Let $\operatorname{Lec}(P ; X)$ be Lecacheux's polynomial with respect to $P$ as in Definition 1.3 with the splitting field $\operatorname{Spl}_{\mathbf{Q}}(\operatorname{Lec}(P ; X))$ over $\mathbf{Q}$.

(i) For any $\mathbf{Q}$-rational point $P \in \mathcal{E}_{p, r}(\mathbf{Q}), \operatorname{Lec}(P ; X)$ is reducible over $\mathbf{Q}$ if and only if $P \in \nu^{*}\left(\mathcal{E}_{p, r}^{*}(\mathbf{Q})\right)$;

(ii) There exists a bijection between the following two finite sets

$$
\left\{\text { subgroup of order } 5 \text { in } \mathcal{E}_{p, r}(\mathbf{Q}) / \nu^{*}\left(\mathcal{E}_{p, r}^{*}(\mathbf{Q})\right)\right\}
$$

and

$$
\left\{\operatorname{Spl}_{\mathbf{Q}}(\operatorname{Lec}(P ; X)) \mid P \in \mathcal{E}_{p, r}(\mathbf{Q}) \backslash \nu^{*}\left(\mathcal{E}_{p, r}^{*}(\mathbf{Q})\right)\right\} .
$$

The bijection is induced by the correspondence $\mathcal{E}_{p, r}(\mathbf{Q}) \ni P \mapsto \operatorname{Spl}_{\mathbf{Q}}(\operatorname{Lec}(P ; X))$.

2. Constructions of $\operatorname{Bru}(t, s ; X)$ and $\operatorname{Lec}(\boldsymbol{p}, \boldsymbol{r} ; \boldsymbol{X})$. We recall constructions of Brumer's polynomial $\mathrm{Bru}(t, s ; X)$ and Lecacheux's polynomial $\operatorname{Lec}(p, r ; X)$ in Lecacheux [7, pages 209-214].

2.1. Construction of $\operatorname{Bru}(t, s ; X)$. We consider the elliptic curve:

$$
E_{t}^{*}: y^{2}+(1-t) x y-t y=x^{3}-t x^{2}
$$

with 5 -torsion points

$$
A=(0,0), 2 A=\left(t, t^{2}\right), 3 A=(t, 0), 4 A=(0, t) .
$$

The curve $E_{t}^{*}$ is also called Tate normal form (see Husemöller [3, page 93, Definition 4.1]). The $j$-invariant of $E_{t}^{*}$ is $\frac{\left(t^{4}-12 t^{3}+14 t^{2}+12 t+1\right)^{3}}{t^{5}\left(t^{2}-11 t-1\right)}$. There exists the elliptic curve $E_{t}=E_{t}^{*} /\langle A\rangle$ up to isomorphism with the isogeny $\phi: E_{t}^{*} \rightarrow E_{t}, X=\frac{t}{x} \mapsto X^{\prime}=$ $\frac{2(X-2)\left(X^{2}+2 X t-1\right)\left(2 X^{2}-2 X t-2 X+t\right)}{X(X-1)^{2}}$ of degree 5 . Then by solving this for $X$, we have $X^{5}+(t-3) X^{4}+$ $\left(1-\frac{1}{4} X^{\prime}-2 t^{2}-\frac{7}{2} t\right) X^{3}+\left(4 t+3+5 t^{2}+\frac{1}{2} X^{\prime}\right) X^{2}+$ $\left(-2 t^{2}-2-\frac{1}{4} X^{\prime}-\frac{5}{2} t\right) X+t=0$. Define $s=-2 t^{2}-$ $2-\frac{1}{4} X^{\prime}-\frac{5}{2} t$. Then the left-hand side of this equation becomes

$$
\begin{aligned}
\operatorname{Bru}(t, s ; x)= & x^{5}+(t-3) x^{4}-(t-s-3) x^{3} \\
& +\left(t^{2}-t-2 s-1\right) x^{2}+s x+t .
\end{aligned}
$$

We find that the elliptic curve $E_{t}$ and the elliptic curve $E_{t, s}$ associated to $\operatorname{Bru}(t, s ; X)$ as in (3) are isomorphic over some extension field (see also Kida, Rikuna and Sato [6, page 695]). The $j$-invariants of $E_{t}$ and of $E_{t, s}$ are the same $\frac{\left(t^{4}+228 t^{3}+494 t^{2}-228 t+1\right)^{3}}{t\left(t^{2}-11 t-1\right)^{5}}$.

2.2. Construction of $\operatorname{Lec}(p, r ; X)$. We consider the elliptic curve

$$
\begin{aligned}
\mathcal{E}_{p}^{*} & : y^{2}-\frac{1}{4}\left(p^{2}+4\right)\left(x^{2}+1\right) \\
& =\frac{1}{2}\left(x^{2}-p x-1\right)(2 x-p)
\end{aligned}
$$

with 5 -torsion points

$$
\begin{aligned}
& A=(\alpha, \beta), 2 A=\left(-\frac{1}{\alpha}, \frac{\beta}{\alpha}\right), \\
& 3 A=\left(-\frac{1}{\alpha},-\frac{\beta}{\alpha}\right), 4 A=(\alpha,-\beta)
\end{aligned}
$$

where $\alpha$ and $-1 / \alpha$ are roots of $x^{2}-p x-1$ and $\beta$ satisfies

$$
\beta^{2}=\frac{1}{4}\left(p^{2}+4\right)\left(\alpha^{2}+4\right)=\frac{1}{4}\left(p^{2}+4\right)^{\frac{3}{2}} \alpha .
$$

The $j$-invariant of $\mathcal{E}_{p}^{*}$ is $\frac{\left(p^{2}-12 p+16\right)^{3}}{p-11}$. There exists the elliptic curve $\mathcal{E}_{p}=\mathcal{E}_{p}^{*} /\langle A\rangle$ up to isomorphism with the isogeny

$$
\begin{aligned}
\phi: \mathcal{E}_{p}^{*} \rightarrow \mathcal{E}_{p}, x \mapsto r= & \frac{x+2 p}{p^{2}+4}+\frac{\left(p^{2}+4\right)(p x+2)}{L^{2}} \\
& +\frac{x(p+2)+\left(p^{2}-p+6\right)}{L}-\frac{5 p}{2\left(p^{2}+4\right)}
\end{aligned}
$$

of degree 5 where $L=x^{2}-p x-1$. Define $l=$ $-L /\left(p^{2}+4\right)$. Solving the equation for $l$, we have

$$
\begin{aligned}
l^{5} & +\left(r^{2}\left(p^{2}+4\right)-2 p-\frac{17}{4}\right) l^{4} \\
& +\left(3 r\left(p^{2}+4\right)+p^{2}+\frac{13}{2} p+5\right) l^{3} \\
& -\left(r\left(p^{2}+4\right)+\frac{11}{2} p-8\right) l^{2}+(p-6) l+1=0 .
\end{aligned}
$$

The left-hand side of this equation yields $\operatorname{Lec}(p, r ; l)$. The elliptic curve $\mathcal{E}_{p}$ and the associated elliptic curve $\mathcal{E}_{p, r}$ to $\operatorname{Lec}(p, r ; X)$ as in (7) are isomorphic over some extension field with the $j$-invariant $\frac{\left(p^{2}+228 p+496\right)^{3}}{(p-11)^{5}}$.

3. Proof of Theorem 1.4. The idea of the proof of Theorem 1.4 is to combine the results given in Hoshi and Miyake [2] and Kida, Rikuna and Sato [6]. According to [2, page 1078, Equation (25)], for $p, r \in \mathbf{Q}$, we define $k=\mathbf{Q}\left(\sqrt{p^{2}+4}\right)$ and 
(8) $s=-\frac{1}{4}\left(5 p+8 r+2 p^{2} r+(2 p r+5) \sqrt{p^{2}+4}\right)$,

$$
t=\frac{1}{2}\left(p+\sqrt{p^{2}+4}\right) .
$$

Then it follows that $\operatorname{Spl}_{k}(\operatorname{Bru}(t, s ; X))=$ $\operatorname{Spl}_{\mathbf{Q}}(\operatorname{Lec}(p, r ; X))$. The associated elliptic curves $E_{t, s}$ and $E_{t, s}^{*}$ given as in (3) and (4) are defined over $k$. According to [6, Section 3], we take elliptic curves $E_{t}$ and $E_{t}^{*}$ defined over $k$ by

$$
\begin{aligned}
E_{t}: & y^{2}-(t-1) x y-t y=x^{3}-t x^{2} \\
& \quad-5 t\left(t^{2}+2 t-1\right) x \\
& \quad t\left(t^{4}+10 t^{3}-5 t^{2}+15 t-1\right) \\
E_{t}^{*}: & y^{2}-(t-1) x y-t y=x^{3}-t x^{2} .
\end{aligned}
$$

The curves $E_{t, s}$ (resp. $E_{t, s}^{*}$ ) and $E_{t}\left(\right.$ resp. $\left.E_{t}^{*}\right)$ are isomorphic over $F=k\left(\sqrt{d_{t, s}}\right)$ where $d_{t, s}$ is given in (2) and we take an isogeny $\phi: E_{t, s} \rightarrow E_{t, s}^{*}$ and the dual isogeny $\phi^{*}: E_{t, s}^{*} \rightarrow E_{t, s}$. We also take an isogeny $\lambda^{*}: E_{t}^{*} \rightarrow E_{t}$ of degree 5 . By $[6$, Theorem 3.1], there exists an injective homomorphism

$$
\begin{aligned}
& E_{t, s}(k) / \phi^{*}\left(E_{t, s}^{*}(k)\right) \\
& \quad \hookrightarrow \operatorname{Hom}_{\operatorname{cont}}\left(\operatorname{Gal}(\bar{F} / F), \operatorname{Ker} \lambda^{*}(k)\right) .
\end{aligned}
$$

We will prove that there exists an injective homomorphism

$$
\begin{aligned}
& \mathcal{E}_{p, r}(\mathbf{Q}) / \nu^{*}\left(\mathcal{E}_{p, r}^{*}(\mathbf{Q})\right) \\
& \quad \hookrightarrow \operatorname{Hom}_{\text {cont }}\left(\operatorname{Gal}(\bar{F} / F), \operatorname{Ker} \lambda^{*}(k)\right) .
\end{aligned}
$$

We see that the elliptic curves $\mathcal{E}_{p, r}$ and $E_{t, s}$ are isomorphic over $k$ with $j$-invariant $\frac{\left(p^{2}+228 p+496\right)^{3}}{(p-1)^{5}}$. Indeed, we may find an isomorphism $f: \mathcal{E}_{p, r}^{(p-11)^{5}} E_{t, s}$ which is given explicitly as

$$
(x, y) \mapsto(a x+b, u y)
$$

where $a, b, u \in k$ are given by

$$
\begin{aligned}
a= & \frac{1}{8}\left(p^{4}+4 p^{2}+2+p\left(p^{2}+2\right) \sqrt{p^{2}+4}\right), \\
b= & \frac{5}{4}\left(p\left(p^{2}+2\right)\left(p^{2}+4\right)\right. \\
& \left.+\left(p^{4}+4 p^{2}+2\right) \sqrt{p^{2}+4}\right) W_{p, r}, \\
u= & \frac{1}{16}\left(\left(p^{2}+2\right)\left(p^{4}+4 p^{2}+1\right)\right. \\
& \left.+p\left(p^{2}+1\right)\left(p^{2}+3\right) \sqrt{p^{2}+4}\right)
\end{aligned}
$$

with $\quad W_{p, r}=16\left(p^{2}+4\right) r^{3}+4\left(p^{2}+4\right) r^{2}-4(19 p+$ 41) $r-16 p-199$ given as in (6).

We obtain an isomorphism $f^{*}: \mathcal{E}_{p, r}^{*} \rightarrow E_{t, s}^{*}$ defined over $k$ such that the diagram

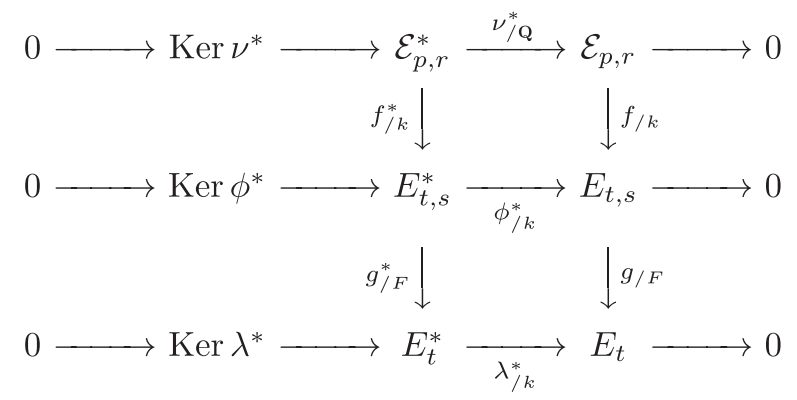

commutes with exact rows. The $j$-invariants of $\mathcal{E}_{p, r}^{*}$ and $E_{t, s}^{*}$ are the same $\frac{\left(p^{2}-12 p+16\right)^{3}}{p-11}$. Therefore the isomorphism $f$ induces an injection

$$
\bar{f}: \mathcal{E}_{p, r}(k) / \nu^{*}\left(\mathcal{E}_{p, r}^{*}(k)\right) \hookrightarrow E_{t, s}(k) / \phi^{*}\left(E_{t, s}^{*}(k)\right) .
$$

By [6, Theorem 3.1] (see also Kida [5, Remark 4.3]), there exists an injective homomorphism

$$
\begin{aligned}
\bar{g}: & E_{t, s}(k) / \phi^{*}\left(E_{t, s}^{*}(k)\right) \\
& \hookrightarrow \operatorname{Hom}_{\operatorname{cont}}\left(\operatorname{Gal}(\bar{F} / F), \operatorname{Ker} \lambda^{*}(k)\right) .
\end{aligned}
$$

Then we also obtain an injective homomorphism

$$
\begin{aligned}
& \bar{g} \circ \bar{f}: \mathcal{E}_{p, r}(k) / \nu^{*}\left(\mathcal{E}_{p, r}^{*}(k)\right) \\
& \hookrightarrow \operatorname{Hom}_{\operatorname{cont}}\left(\operatorname{Gal}(\bar{F} / F), \operatorname{Ker} \lambda^{*}(k)\right) .
\end{aligned}
$$

Because the isogeny $\nu^{*}$ is defined over $\mathbf{Q}$, we get

$$
\begin{aligned}
& \mathcal{E}_{p, r}(\mathbf{Q}) / \nu^{*}\left(\mathcal{E}_{p, r}^{*}(\mathbf{Q})\right) \\
& \quad \hookrightarrow \operatorname{Hom}_{\operatorname{cont}}\left(\operatorname{Gal}(\bar{F} / F), \operatorname{Ker} \lambda^{*}(k)\right) .
\end{aligned}
$$

Every point $P=(x(P), y(P)) \in \mathcal{E}_{p, r}(\mathbf{Q})$ defines a Kummer extension

$$
L_{P}=F\left(\left(\lambda^{*}\right)^{-1}(g \circ f(P))\right)
$$

over $F$. In particular, via (8), we observe that

$$
\begin{aligned}
L_{P} & =\operatorname{Spl}_{k}\left(\operatorname{Bru}\left(\frac{1}{2}\left(p+\sqrt{p^{2}+4}\right), \frac{x(f(P))}{-4 d} ; X\right)\right) \\
& =\operatorname{Spl}_{\mathbf{Q}}(\operatorname{Lec}(P ; X))
\end{aligned}
$$

where $\operatorname{Lec}(P ; X)=\operatorname{Lec}\left(p, \frac{x(P)}{4\left(p^{2}+4\right) W} ; X\right)$ as in Definition 1.3. Hence the group $\mathcal{E}_{p, r}(\mathbf{Q}) / \nu^{*}\left(\mathcal{E}_{p, r}^{*}(\mathbf{Q})\right)$ classifies the isomorphism classes of $\operatorname{Spl}_{\mathbf{Q}}(\operatorname{Lec}(P ; X))$ with quartic subfield $F$ (see also [6, Section 3]).

By Theorem 1.4, we have the following result by the multiplication-by-2 map of the elliptic curve $\mathcal{E}_{p, r}$ :

Corollary 3.1. For a $\mathbf{Q}$-rational point $P \in$ $\mathcal{E}_{p, r}(\mathbf{Q})$ and integer $n$ with $\operatorname{gcd}(n, 5)=1$, $\operatorname{Spl}_{\mathbf{Q}}(\operatorname{Lec}(P ; X))=\operatorname{Spl}_{\mathbf{Q}}(\operatorname{Lec}([n] P ; X)) \quad$ where $\operatorname{Lec}(P ; X)=\operatorname{Lec}\left(p, \frac{x(P)}{4\left(p^{2}+4\right) W} ; X\right)$ as in Definition 1.3. 
In particular, for $P=Q_{0}=\left(4 r\left(p^{2}+4\right) W, 2\left(p^{2}+\right.\right.$ 4) $\left.W^{2}\right)$ and $n=2$, we have $\operatorname{Spl}_{\mathbf{Q}}(\operatorname{Lec}(p, r ; X))=$ $\operatorname{Spl}_{\mathbf{Q}}(\operatorname{Lec}(p, R ; X))$ where

$$
\begin{aligned}
R= & \frac{x\left([2] Q_{0}\right)}{4\left(p^{2}+4\right) W}, \\
W= & 16\left(p^{2}+4\right) r^{3}+4\left(p^{2}+4\right) r^{2} \\
& -4(19 p+41) r-16 p-199, \\
x\left([2] Q_{0}\right)= & 16\left(p^{2}+4\right)^{2} r^{4}+8\left(p^{2}+4\right)(19 p+41) r^{2} \\
& +4\left(32 p^{3}+398 p^{2}+128 p+1592\right) r \\
& +16 p^{3}+560 p^{2}+1622 p+2477 .
\end{aligned}
$$

Remark 3.2. We can also verify that $\operatorname{Spl}_{\mathbf{Q}}(\operatorname{Lec}(p, r ; X))=\operatorname{Spl}_{\mathbf{Q}}(\operatorname{Lec}(p, R ; X))$ in Corollary 3.1 by Hoshi and Miyake [2] via multi-resolvent polynomials. We take multi-resolvent polynomials $F_{a, a^{\prime}}^{1}$ and $F_{a, a^{\prime}}^{2}$ as in [2, page 1071] where $a=(s, t), a^{\prime}=\left(s^{\prime}, t^{\prime}\right)$. Using [2, page 1078, Method $2]$, via $(8)$, we obtain that $\operatorname{Spl}_{\mathbf{Q}}(\operatorname{Lec}(p, r ; X))=$ $\mathrm{Spl}_{\mathbf{Q}}(\operatorname{Lec}(p, R ; X))$ if and only if $F_{a, a^{\prime}}^{1}$ or $F_{a, a^{\prime}}^{2}$ has a linear factor over $k=\mathbf{Q}\left(\sqrt{p^{2}+4}\right)$. Indeed, we see that $F_{a, a^{\prime}}^{2}$ has a linear factor $x+\frac{1+2 r}{2} \sqrt{p^{2}+4}+\frac{p-1}{2}$.

4. Examples of Theorem 1.4. We will give two examples of Theorem 1.4.

Example $4.1(p=1$ and $r=-3$ with $\left.\mathcal{E}_{1,-3}(\mathbf{Q}) / \nu^{*}\left(\mathcal{E}_{1,-3}^{*}(\mathbf{Q})\right) \simeq \mathbf{Z} / 5 \mathbf{Z}\right)$. We consider the case where $p=1$ and $r=-3$. The associated isogenous curves are

$$
\begin{aligned}
\mathcal{E}_{1,-3}: y^{2}= & x^{3}-7375 x^{2}-2610750000 x \\
& +68994507812500 \\
\mathcal{E}_{1,-3}^{*}: y^{2}= & x^{3}-7375 x^{2}-11313250000 x \\
& -5450566117187500
\end{aligned}
$$

with $j$-invariants $-\frac{5 \cdot 29^{3}}{2^{5}},-\frac{5^{2}}{2}$ respectively. Their Mordell-Weil groups are

$$
\begin{aligned}
& \mathcal{E}_{1,-3}(\mathbf{Q})=\left\langle P_{1}, P_{2}\right\rangle \simeq \mathbf{Z}^{\oplus 2}, \\
& \mathcal{E}_{1,-3}^{*}(\mathbf{Q})=\left\langle Q_{1}, Q_{2}\right\rangle \simeq \mathbf{Z}^{\oplus 2}
\end{aligned}
$$

where

$$
\begin{aligned}
P_{1} & =(-53100,6091750), \\
P_{2} & =(88500,21756250), \\
Q_{1} & =(678500,543906250), \\
Q_{2} & =(1452875,1740500000) .
\end{aligned}
$$

We see $P_{2}=Q_{0}$ where $Q_{0}=\left(4 r\left(p^{2}+4\right) W, 2\left(p^{2}+\right.\right.$ 4) $\left.W^{2}\right)$ which corresponds to $\operatorname{Lec}(1,-3 ; X)$. The isogeny $\nu^{*}: \mathcal{E}_{1,-3}^{*} \rightarrow \mathcal{E}_{1,-3}$ is given by

$$
\begin{aligned}
& \nu^{*}\left(Q_{1}\right)=P_{1}-2 P_{2}, \\
& \nu^{*}\left(Q_{2}\right)=-P_{1}-3 P_{2} .
\end{aligned}
$$

Hence the image of $\nu^{*}$ is given by

$$
\nu^{*}\left(\mathcal{E}_{1,-3}^{*}\right)=\left\langle P_{1}-2 P_{2}, 5 P_{2}\right\rangle .
$$

We conclude that $\mathcal{E}_{1,-3}(\mathbf{Q}) / \nu^{*}\left(\mathcal{E}_{1,-3}^{*}(\mathbf{Q})\right)=\left\langle\overline{P_{2}}\right\rangle \simeq$ $\mathbf{Z} / 5 \mathbf{Z}$. Thus there exists exactly one isomorphism class of Lecachux's polynomials. We have

$$
\begin{aligned}
\operatorname{Spl}_{\mathbf{Q}}(\operatorname{Lec}(1,-3 ; X)) & =\operatorname{Spl}_{\mathbf{Q}}\left(\operatorname{Lec}\left([n] P_{2} ; X\right)\right) \\
& =\operatorname{Spl}_{\mathbf{Q}}\left(\operatorname{Lec}\left(1, \frac{x\left([n] P_{2}\right)}{4\left(p^{2}+4\right) W} ; X\right)\right)
\end{aligned}
$$

where $\operatorname{gcd}(n, 5)=1$. For example, for $n=1,2,3,4$, we have

$$
\frac{x\left([n] P_{2}\right)}{4\left(p^{2}+4\right) W}=-3, \frac{-263}{236}, \frac{4849}{39605}, \frac{2034016227}{1036798976}
$$

respectively. We can check this example by Sage [9] as in the arXiv version of this paper [1, Example 4.1].

Example $4.2(p=2$ and $r=-15 \quad$ with $\left.\mathcal{E}_{2,-15}(\mathbf{Q}) / \nu^{*}\left(\mathcal{E}_{2,-15}^{*}(\mathbf{Q})\right) \simeq(\mathbf{Z} / 5 \mathbf{Z})^{\oplus 2}\right)$. We consider the case where $p=2, r=-15$. The associated isogenous curves are

$$
\begin{aligned}
\mathcal{E}_{2,-15}: y^{2}= & x^{3}-3362328 x^{2}-446557358393568 x \\
& +4390381057572915584256
\end{aligned}
$$

$\mathcal{E}_{2,-15}^{*}: y^{2}=x^{3}-3362328 x^{2}+1181398581066528 x$ - 243295532112514685688576

with $j$-invariants $-\frac{2^{6} \cdot 239^{3}}{3^{10}}, \frac{2^{6}}{3^{2}}$ respectively. Their Mordell-Weil groups are

$$
\begin{aligned}
& \mathcal{E}_{2,-15}(\mathbf{Q})=\left\langle P_{\text {tor }}\right\rangle \oplus\left\langle P_{1}, P_{2}, P_{3}\right\rangle \simeq \mathbf{Z} / 2 \mathbf{Z} \oplus \mathbf{Z}^{\oplus 3}, \\
& \mathcal{E}_{2,-15}^{*}(\mathbf{Q})=\left\langle Q_{\text {tor }}\right\rangle \oplus\left\langle Q_{1}, Q_{2}, Q_{3}\right\rangle \simeq \mathbf{Z} / 2 \mathbf{Z} \oplus \mathbf{Z}^{\oplus 3}
\end{aligned}
$$

where

$$
\begin{aligned}
P_{\text {tor }} & =(-23536296,0), \\
P_{1} & =\left(\frac{1213850592}{121}, \frac{32104365187824}{1331}\right), \\
P_{2} & =(12954852,14669441496), \\
P_{3} & =(24185016,75959770464), \\
Q_{\text {tor }} & =(57159576,0), \\
Q_{1} & =\left(\frac{9662338144}{169}, \frac{26786536642000}{2197}\right), \\
Q_{2} & =(58184676,105083001000), \\
Q_{3} & =\left(\frac{15400097496}{121}, \frac{1841522732064000}{1331}\right) .
\end{aligned}
$$

The isogeny $\nu^{*}: \mathcal{E}_{2,-15}^{*} \rightarrow \mathcal{E}_{2,-15}$ is given by

$$
\begin{aligned}
\nu^{*}\left(Q_{\text {tor }}\right) & =P_{\text {tor }}, \\
\nu^{*}\left(Q_{1}\right) & =-P_{1}+2 P_{2}+2 P_{3}, \\
\nu^{*}\left(Q_{2}\right) & =P_{\text {tor }}-2 P_{1}-P_{2}-P_{3}, \\
\nu^{*}\left(Q_{3}\right) & =-2 P_{1}+4 P_{2}-P_{3} .
\end{aligned}
$$

Hence we obtain the image 


$$
\nu^{*}\left(\mathcal{E}_{2,-15}^{*}\right)=\left\langle P_{\text {tor }}, P_{1}+2 P_{2}+2 P_{3}, 5 P_{2}, 5 P_{3}\right\rangle
$$

and conclude that $\mathcal{E}_{2,-15}(\mathbf{Q}) / \nu^{*}\left(\mathcal{E}_{2,-15}^{*}(\mathbf{Q})\right)=$ $\left\langle\overline{P_{2}}, \overline{P_{3}}\right\rangle \simeq(\mathbf{Z} / 5 \mathbf{Z})^{\oplus 2}$. There exist 6 subgroups of order 5 in $\mathcal{E}_{2,-15}(\mathbf{Q}) / \nu^{*}\left(\mathcal{E}_{2,-15}^{*}(\mathbf{Q})\right) \simeq(\mathbf{Z} / 5 \mathbf{Z})^{\oplus 2}$ which correspond to the 6 isomorphism classes

$$
\begin{aligned}
\operatorname{Lec}\left(P_{2}-2 P_{3} ; X\right) & =\operatorname{Lec}\left(2,-\frac{68264085293968884683}{114084259382587016} ; X\right), \\
\operatorname{Lec}\left(P_{2}-P_{3} ; X\right) & =\operatorname{Lec}\left(2,-\frac{5293745}{22271049} ; X\right), \\
\operatorname{Lec}\left(P_{2} ; X\right) & =\operatorname{Lec}\left(2,-\frac{131}{136} ; X\right), \\
\operatorname{Lec}\left(P_{2}+P_{3} ; X\right) & =\operatorname{Lec}\left(2, \frac{157}{559} ; X\right), \\
\operatorname{Lec}\left(P_{2}+2 P_{3} ; X\right) & =\operatorname{Lec}\left(2, \frac{9701177386741}{7753965979144} ; X\right), \\
\operatorname{Lec}\left(P_{3} ; X\right) & =\operatorname{Lec}\left(2,-\frac{19759}{10988} ; X\right),
\end{aligned}
$$

with the quartic subfield

$$
F=\mathbf{Q}\left(\sqrt{-233495-\frac{326893}{2} \sqrt{2}}\right) .
$$

Since Lec $(2,-15 ; X)$ corresponds to the point

$$
\begin{aligned}
& Q_{0}=\left(4 r\left(p^{2}+4\right) W, 2\left(p^{2}+4\right) W^{2}\right) \\
& =(201739680,2826312394896)=P_{\text {tor }}-P_{1}-P_{3} \\
& \text { and }\left\langle\overline{Q_{0}}\right\rangle=\left\langle\overline{P_{2}-2 P_{3}}\right\rangle \text { in } \mathcal{E}_{2,-15}(\mathbf{Q}) / \nu^{*}\left(\mathcal{E}_{2,-15}^{*}(\mathbf{Q})\right), \\
& \operatorname{Spl}_{\mathbf{Q}}\left(\operatorname{Lec}\left(Q_{0} ; X\right)\right)=\operatorname{Spl}_{\mathbf{Q}}(\operatorname{Lec}(2,-15 ; X)) \\
& =\operatorname{Spl}_{\mathbf{Q}}\left(\operatorname{Lec}\left(P_{2}-2 P_{3} ; X\right)\right) .
\end{aligned}
$$

We can check this example by Sage [9] as in the arXiv version of this paper [1, Example 4.2].

Two examples of the degenerate cases $G_{p, r} \simeq$ $D_{5}$ and $C_{5}$ where $G_{p, r}=\operatorname{Gal}(\operatorname{Lec}(p, r ; X) / \mathbf{Q})$ are also given in [1, Section 5].
Acknowledgments. The authors thank the referee for helpful comments. This work was partially supported by JSPS KAKENHI Grant Number 19K03418.

\section{References}

[ 1 ] A. Hoshi and M. Koshiba, On Lecacheux's family of quintic polynomials, arXiv:2003.13458 (the arXiv version of this paper)

[ 2 ] A. Hoshi and K. Miyake, On the field intersection problem of solvable quintic generic polynomials, Int. J. Number Theory 6 (2010), no. 5, 1047-1081.

[ 3 ] D. Husemöller, Elliptic curves, 2nd ed., Graduate Texts in Mathematics, 111, Springer-Verlag, New York, 2004.

[ 4 ] C. U. Jensen, A. Ledet and N. Yui, Generic polynomials, Mathematical Sciences Research Institute Publications, 45, Cambridge University Press, Cambridge, 2002.

[ 5 ] M. Kida, On metacyclic extensions, J. Théor. Nombres Bordeaux 24 (2012), no. 2, 339-353.

[ 6 ] M. Kida, Y. Rikuna and A. Sato, Classifying Brumer's quintic polynomials by weak MordellWeil groups, Int. J. Number Theory 6 (2010), no. 3, 691-704.

[ 7 ] O. Lecacheux, Constructions de polynômes génériques à groupe de Galois résoluble, Acta Arith. 86 (1998), no. 3, 207-216.

[ 8 ] J. H. Silverman, The arithmetic of elliptic curves, Graduate Texts in Mathematics, 106, SpringerVerlag, New York, 1986.

[ 9 ] W. A. Stein, et al., Sage: Open Source Mathematical Software (Version 9.0), The Sage Group, http://www.sagemath.org, 2020.

[ 10 ] J. Vélu, Isogénies entre courbes elliptiques, C. R. Acad. Sci. Paris Sér. A-B 273 (1971), A238A241. 